\title{
Study of oxygen content in titanium alloys after exposure at elevated temperature
}

\author{
M. Kalienko ${ }^{1,2^{*}}$, A. Volkov ${ }^{1}$, M. Leder ${ }^{1}$, A. Zhelnina ${ }^{1}$ \\ ${ }^{1}$ PSC VSMPO-AVISMA Corporation, Parkovaya st. 1, Verkhnaya Salda 624760, Russia \\ 2 Ural Federal University, Mira st. 19, Ekaterinburg 620002, Russia \\ Email*, kalienko@vsmpo.ru
}

\begin{abstract}
Isothermal oxidation testing of near a titanium alloys VT18U, VT20, Ti6AI7Nb and Ti6242S was performed in air at $560{ }^{\circ} \mathrm{C}$ for 1000 hours. Parameters of diffusion layer on the alloy surfaces were studied by microhardness indentations, optical microscopy, $\mathrm{X}$-ray diffraction analysis and nuclear microanalysis. It was established that concentration of oxygen in diffusion layer of tested alloys after oxidation differs significantly. An approach was demonstrated and validated by nuclear microanalysis data that allows comparative evaluation of the total concentration of interstitial impurities in the diffusion layer by the X-ray diffraction method.
\end{abstract}

Keywords: titanium alloys, oxidation, diffusion, lattice parameters, X-ray diffraction analysis.

\section{Introduction}

A breakthrough development of high temperature titanium alloys in 1960-1970s inspired advancement of aircraft- and enginebuilding. Well-known alloys such as IMI685, B120, Ti6242, and Russian alloys VT18 and VT20 have been developed during this time period [1-6]. The new high temperature titanium alloys allowed creation of new aerospace technologies, e.g. fully 93 percent of the legendary Blackbird's structural weight consisted of titanium alloys [7]. This aircraft was designed and put into operation in 1960s.

The maximum operating temperature of near a titanium alloys is limited to $600{ }^{\circ} \mathrm{C}$. Higher temperatures drastically reduce high temperature strength, creep resistance [1, 3, 5]. Moreover, long-term exposure of titanium alloys to air at temperatures over 400 ${ }^{\circ} \mathrm{C}$ leads to oxidezes and formation diffusion layer due to of high sulubility of oxygen in the matrix [8-10]. The resultant diffusion layer is characterized by high strength, low ductility and toughness, it significantly reduces fatigue strength. It has been shown that high temperature alloys IMI834, Ti6242S, Beta21S, and Ti1100 exhibited deteriorated ductility and fatigue strength after operation at elevated temperatures, when tested in the oxidized state [10-15].

At the moment, there are still some open issues concerning relationship between the main parameters influencing the metal (temperature, time, atmosphere, pressure) with its structural characteristics (oxygen concentration in metal, gas-rich layer thickness, material hardness, thickness, density and oxide adhesion, weight gain, diffusion coefficients, etc.) and mechanical properties in the oxidized state (fatigue strength, ductility, tensile strength). Therefore, further study of air oxidation behavior of titanium alloys is a vital scientific and engineering task. The objective of this work is to demonstrate new data that facilitates better understanding of the nature of deterioration of ductility and other mechanical properties after exposure of titanium alloys at elevated temperatures.

\section{Material and experiments}

The studied materials were sheets made of titanium alloys VT18U, VT20, Ti6AI7Nb and Ti6242S by VSMPO-AVISMA Corporation. Sheets were cut to $20 \times 20 \mathrm{~mm}$ samples using electrical discharge machining, thereafter, all samples were mechanically ground (P240), degreased with petrol and cleaned with ethyl alcohol.

Oxidation of samples was performed in air at $560^{\circ} \mathrm{C}$ for 1000 hours. Oxidation was done in an electric laboratory furnace with a fan for air mixing and temperature maintenance within $\pm 5^{\circ} \mathrm{C}$.

X-ray diffraction analysis (XRD) of samples was performed on a Bruker D8 Advance diffractometer with a LynxEye detector. XRD patterns were taken in copper radiation in the following conditions: tube voltage of $40 \mathrm{kV}$, tube current of $40 \mathrm{~mA}$, step size $0.02^{\circ}$, exposure $0.5 \mathrm{sec}$ per point. The lattice parameters were refined using TOPAS 3 software by a whole pattern modeling in the angle range of 34 to $43^{\circ} 2 \theta$ [16]. The qualitative phase analysis of the annealed samples was performed using crystallographic database PDF2007 and software package EVA13.0.0.3. The diffractometer was aligned using the corundum (NIST SRM1976b).

Concentrations of oxygen and nitrogen near the oxide/metal interface were studied by nuclear microanalysis (NMA) using $2 \mathrm{MW}$ Van de Graaf accelerator EG-2M-1. The concentration profiles of oxygen, nitrogen and carbon atoms were measured via reactions 
${ }^{16} \mathrm{O}\left(\mathrm{d}, \mathrm{p}_{1}\right){ }^{17} \mathrm{O}$ and ${ }^{14} \mathrm{~N}\left(\mathrm{~d}, \mathrm{a}_{1}\right){ }^{12} \mathrm{C}[17,18]$, respectively.

Metallographic analysis and microhardness measurements were performed on samples that were cross-sectioned from the ones for XRD and NMA. Vickers microhardness measurements were made using a diamond square pyramid indenter and 10 gr load by Shtruers Duramin tester. The observation of the surface oxide was performed by scanning electron microscope (SEM) Quanta 3D FEG. To quantify the thickness of diffusion layer, the microsections were etched in $5 \%$ aqueous solution of hydrofluoric acid. The thickness of gas-rich layer was taken as the average thickness of near-surface structure with a lighter contrast compared to the base metal. The obtained thickness values were averaged over 60-70 measurements taken in different places on the sample surface.

\section{Results}

Figure 1 shows a diffusion layer on the oxidized alloy samples after oxidation. The average thickness of oxygen penetration measured on microsections after examination by optical microscopy (OM) is given in Table 1. The minimum thickness of diffusion layer is observed in Ti6Al7Nb, the maximum thickness - in Ti6242S.

Table 1. Thickness of diffusion layer, thickness of oxide scale after oxidation in air at $560^{\circ} \mathrm{C}$ for 1000 hours

\begin{tabular}{|l|c|c|}
\hline & Diffusion layer, microns (OM) & Oxide scale thickness, nm (SEM) \\
\hline Ti6Al7Nb & 14 & 260 \\
\hline VT20 & 20 & 480 \\
\hline Ti6242S & 20 & 280 \\
\hline VT18U & 17 & 280 \\
\hline
\end{tabular}
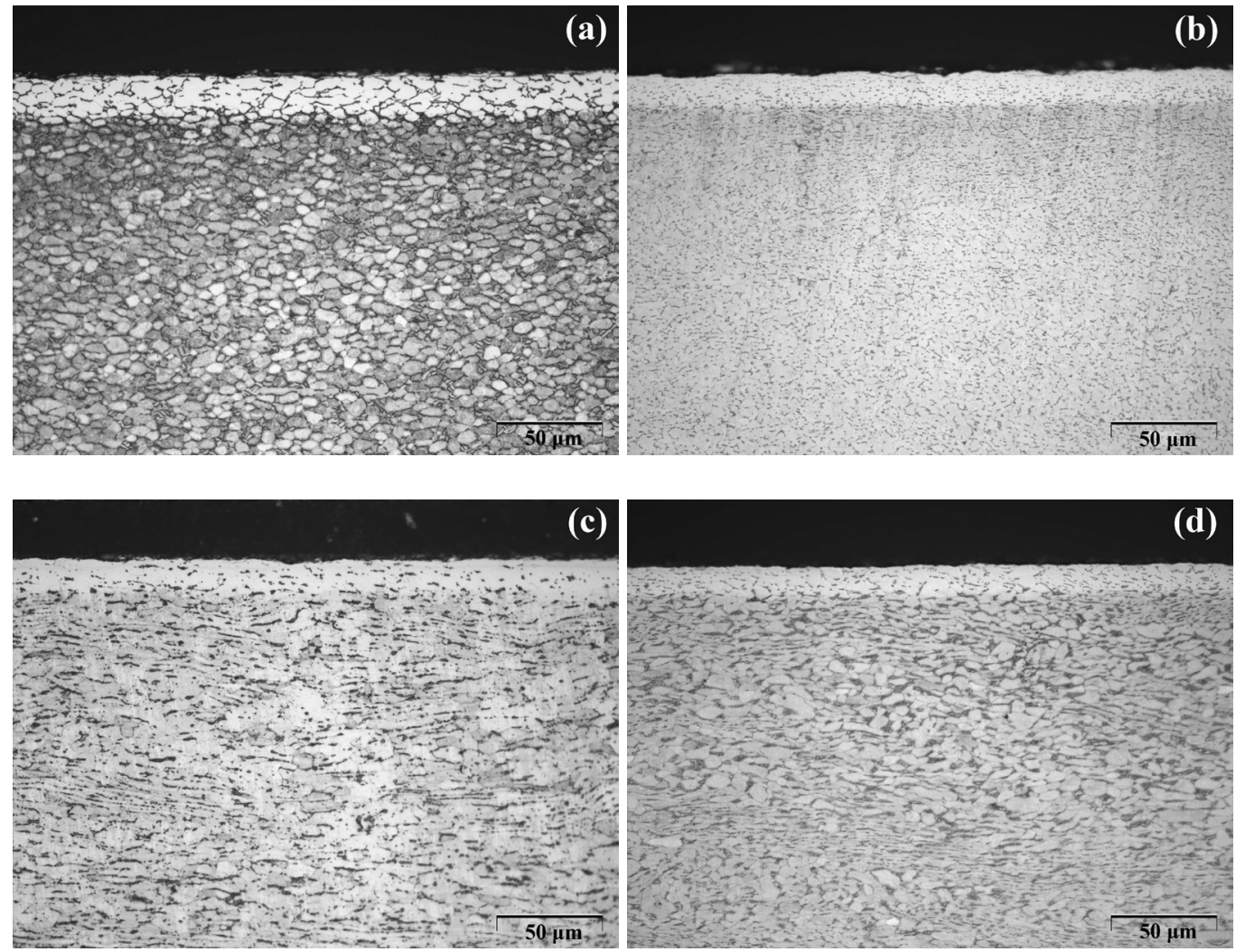

Fig. 1. Optical micrograph showing diffusion layer formed after oxidation in air at $560^{\circ} \mathrm{C}$ for 1000 hours: (a) Ti6242; (b) VT20; (c) VT18U; (d) Ti6Al7Nb

Figure 2 shows the oxide formed on the surface of Ti6242S as a result of oxidation, this oxide has a typical needle-like morphology [19]. The results of qualitative XRD analysis demonstrate that the oxide scale consists of TiO2 rutile type, Fig. 3. 

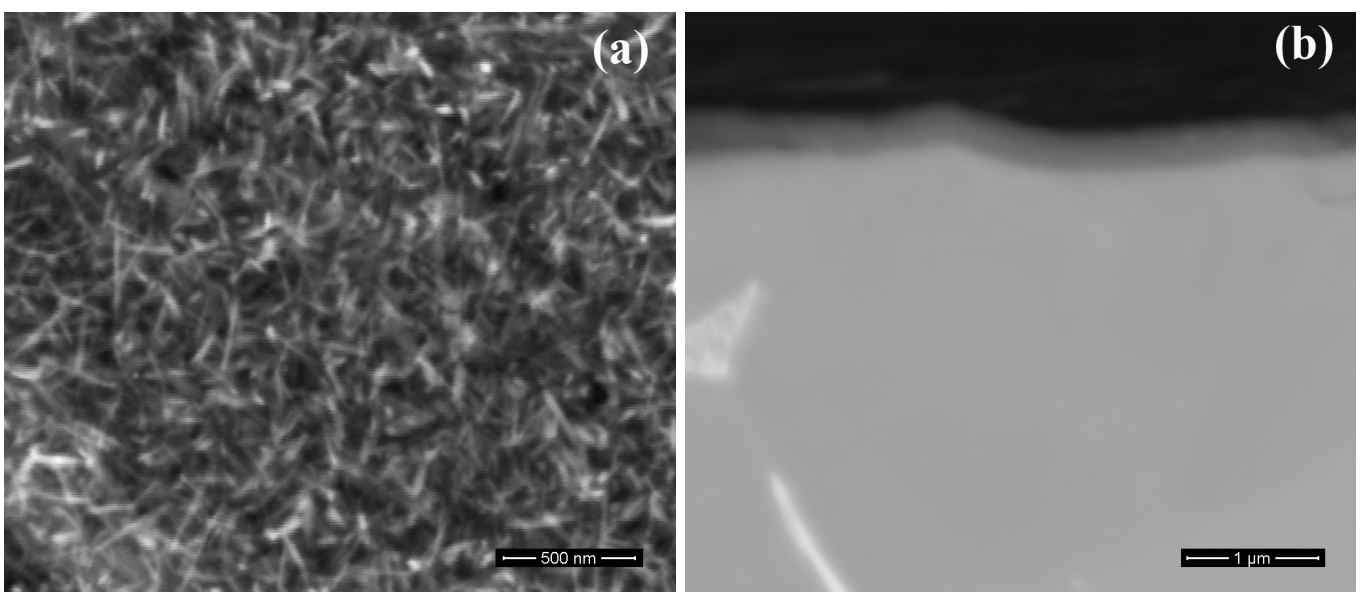

Fig. 2. Oxide on the surface of Ti6242S sample (a) and its cross section (b)

The density of pure rutile is less than that of titanium and is $4.2 \mathrm{~g} / \mathrm{cm}^{2}$ [20]. Alloyed titanium alloys may have varying phase composition and density of the oxide scale due to the presence of alloying elements in the oxide scale [21]. Table 1 presents the data on the thickness of the oxide scale on sample surfaces. The thickness of the oxide scale on the studied samples is less than $500 \mathrm{~nm}$, therefore the X-ray diffraction allows to obtain information on the structure of gas-rich layer and oxide. The calculated depth of copper radiation penetration contributing $80 \%$ to the diffracted radiation is $\approx 3 \mu \mathrm{m}$ at $43^{\circ} 2 \theta$ (soft - AbsorbDX V1.1.4).

Oxygen, nitrogen and carbon are interstitial atoms located in the interstices of the crystal lattice of titanium [22]. During the oxidation of titanium alloys in air, oxygen is the main element that forms a diffusion layer, but we failed to find accurate information on the distribution of gases in the diffusion layers of various titanium alloys. Further to the oxidation theory, oxygen distribution in diffusion layer after annealing has a certain gradient; this gradient depends on the temperature and time of oxidation, and also on the oxide characteristics (presence of defects, phase composition, thickness, etc.), oxygen solubility in the alloy, oxygen concentration near oxide/metal interface, diffusion rate and activation energy [8-10, 22-26].
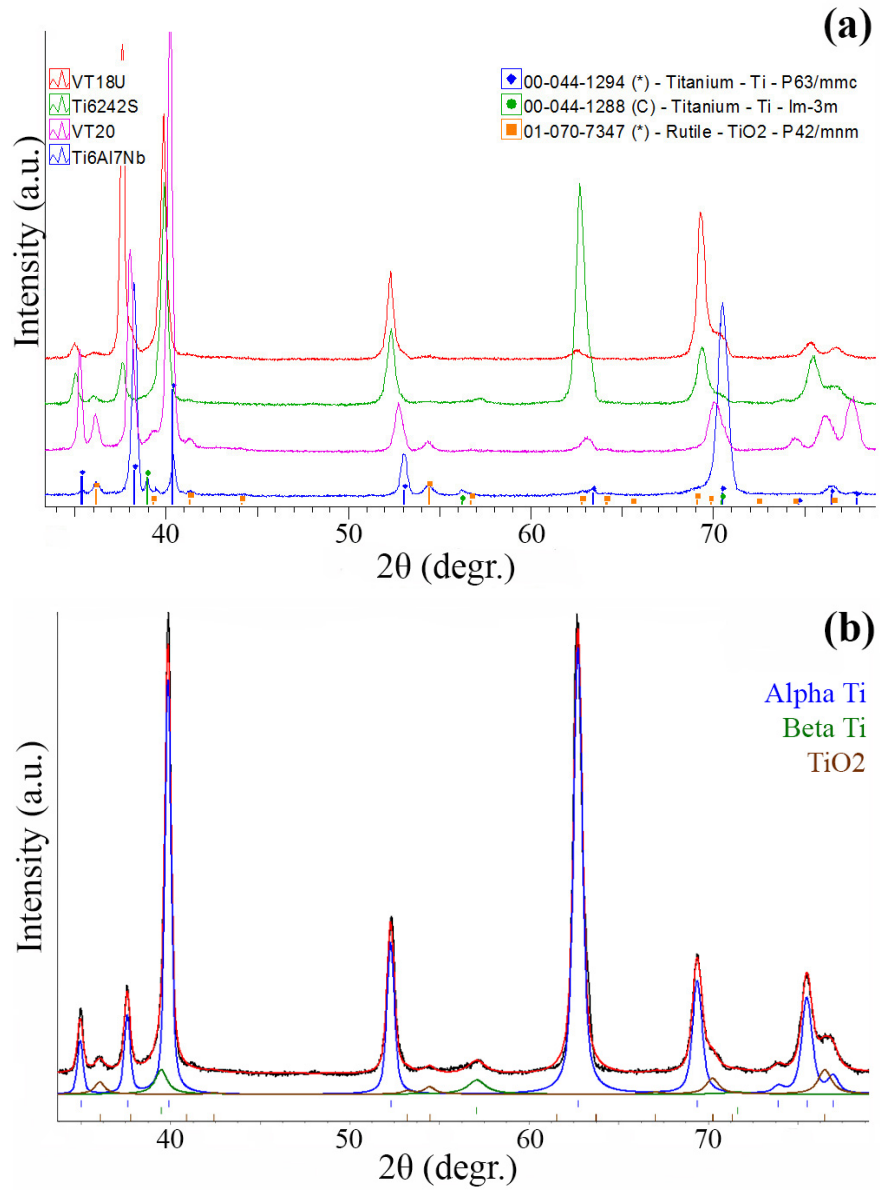

Fig. 3. Diffraction patterns of the alloys after oxidation with markers of identified phases (a) and profile fits of the model diffraction pattern for Ti6242S (b) 
Increasing oxygen concentration leads to the increase of the lattice parameters and thus to the expansion of the volume of titanium unit cell $[22,27]$. Studies [28-30] demonstrate approaches that allow the assessment of oxygen concentration in titanium by the parameters of its crystal lattice. Capability of the XRD analysis of diffusion layer is limited to the depth of X-ray penetration and is substantially complicated by the presence of oxygen concentration gradient, which forms a diffraction pattern resulting from the superposition of the diffracted radiation. Nevertheless, use of the whole pattern modeling of diffraction patterns allows comparative evaluation of oxygen concentration in diffusion layer.

Figure 3 shows diffraction patterns taken from the surface of oxidized alloys, as can be seen, location of lines significantly differs for studied alloys. As mentioned above, increasing oxygen concentration leads to the expansion of the volume of the unit cell, which results in a shift of diffraction lines on the diffraction pattern towards small angles, proportional to the oxygen concentration. Slight asymmetry of diffraction lines is associated with the gradient of oxygen concentration.

The profile of the diffraction pattern (Fig. 3) fitted using a phases of titanium with hexagonal symmetry (P63/mmc), $\beta$ phase of titanium with cubic symmetry $(\mathrm{Im}-3 \mathrm{~m})$ and tetragonal phase of titanium oxide TiO2 $(\mathrm{P} 42 / \mathrm{mnm})$ was used to calculate the lattice parameters of the alloys before and after oxidation. Data on the relative variation of the volume of the unit cell of a phase of titanium $\Delta V=\left(V_{a}-V_{a 0}\right) \times 100 / V_{a 0}$, where $V_{a}$ is the cell volume near the oxide/metal interface after oxidation and $V_{a 0}$ is the initial value for Ti6AI7Nb, VT20, Ti6242S and VT18U alloys: 0.97, 2.07, 3.93 and $4.14 \%$ respectively. Minimum increment of the volume of the unit cell of titanium a phase after oxidation was obtained for Ti6Al7Nb, while maximum increment was obtained for VT18U. The obtained XRD data indirectly characterize the concentration of oxygen in the diffusion layer of the alloys and, as can be seen, is confirmed by the results of nuclear microanalysis, Figure 4 (a). According to the obtained data, the oxygen concentration at $0.5 \mu \mathrm{m}$ from the surface for Ti6AI7Nb, VT20, Ti6242S and VT18U is 9.32, 13.18, 13.95, 14.42 wt \% respectively and decreases towards the metal core approximately with one gradient. Thus, there is a good correlation of XRD and NMA data testifying to the fact that the concentration of oxygen in diffusion layer of studied alloys differs significantly.
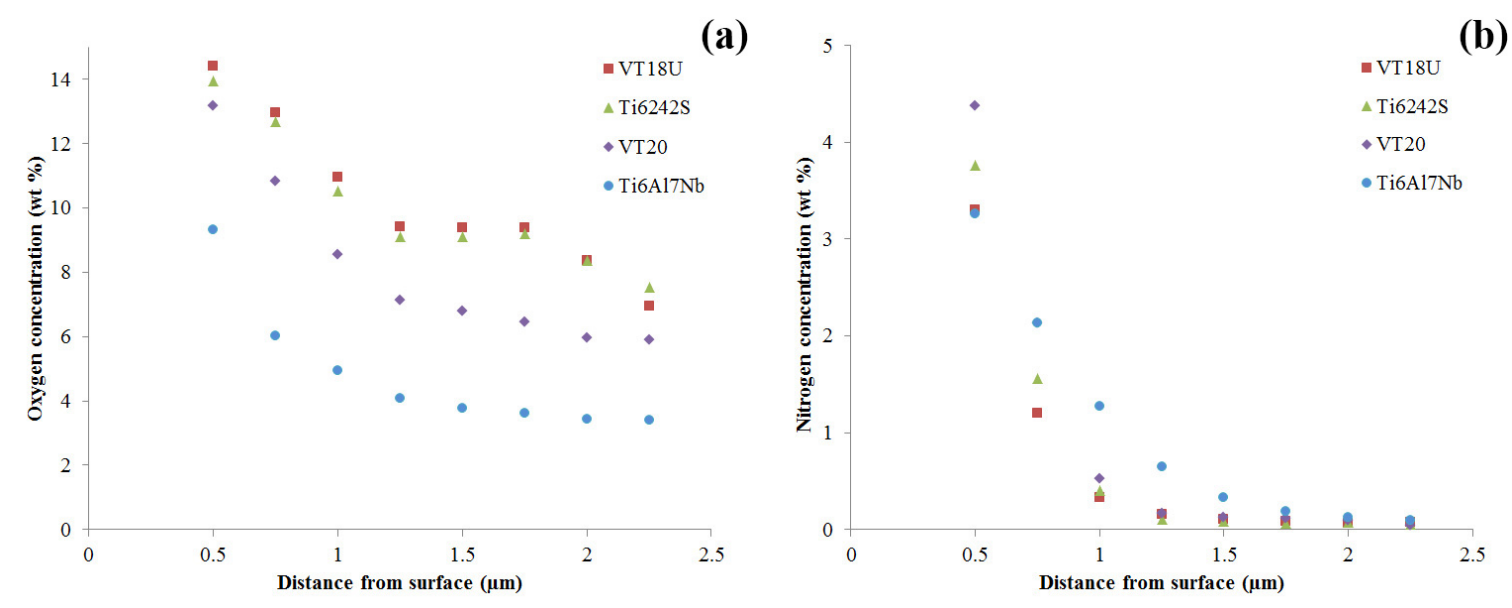

Fig. 4. Distribution of oxygen (a) and nitrogen (b) concentrations near the oxide/metal interface in titanium alloys after oxidation in air at 560 ${ }^{\circ} \mathrm{C}$ for 1000 hours

Figure 4 (b) shows the dependences of nitrogen concentration in diffusion layer; samples all alloys exhibit a very thin layer with the increased concentration of nitrogen near the oxide/metal interface. Formation of this layer was also observed in other studies [31]. Nitrogen concentration decreases to values below $0.1 \mathrm{wt}$. \% in all alloys at of 2.3 microns from the oxide surface. Thus, it is clear that oxygen is the main contributor to the formation of diffusion layer during the oxidation of alloys in air.

Measurement of hardness of diffusion layer is an effective tool for analyzing oxygen gradient in diffusion layer [13, 27, 30]. It is obvious that the observed difference in oxygen concentration near the oxide/metal interface of the studied alloys determines the oxygen gradient in the entire diffusion layer, Fig. 5 (a). Figure 5 (b) shows indentations of various sizes on the studied alloys Ti6242S and Ti6Al7Nb. 

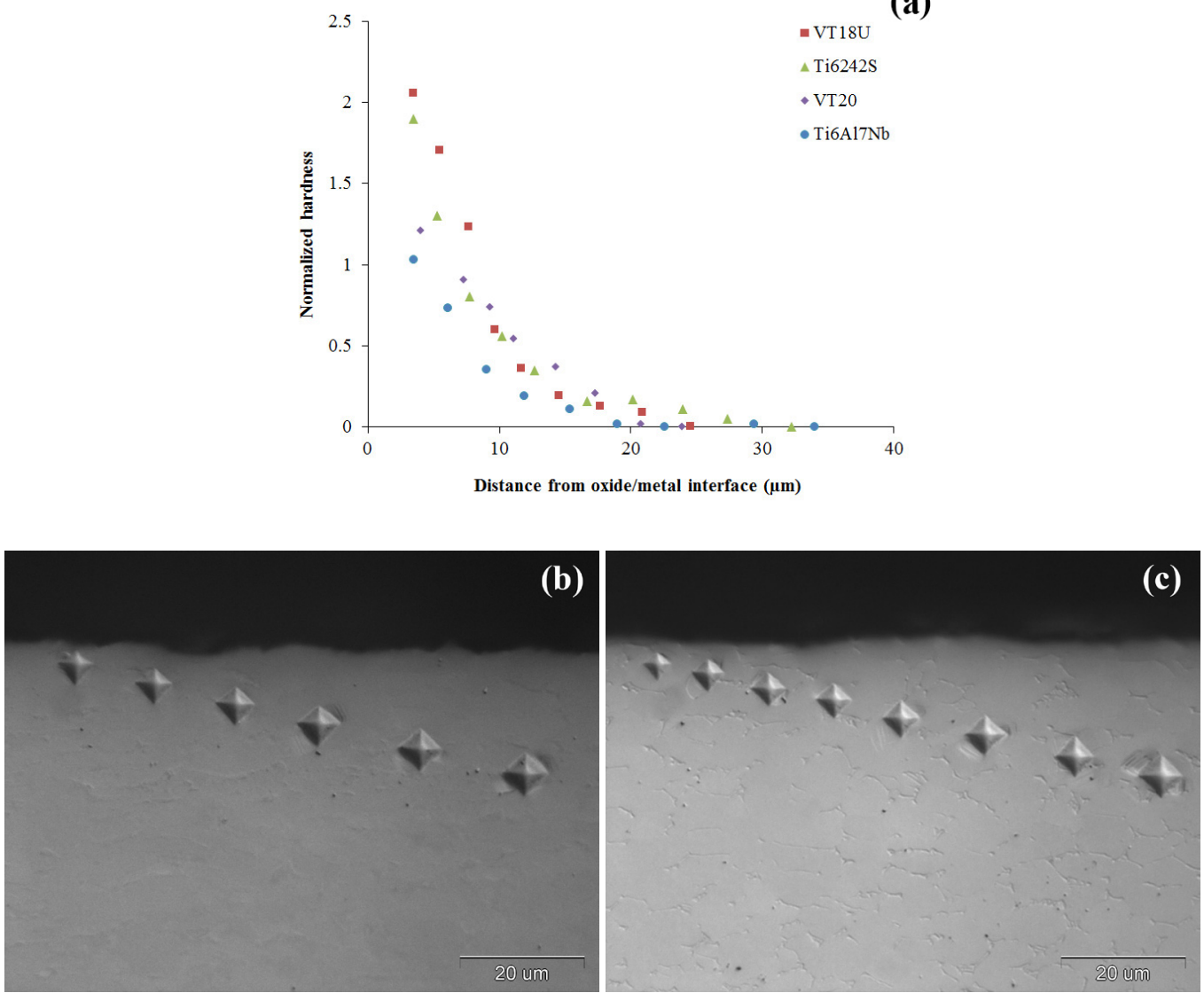

Fig. 5. Normalized hardness increase in diffusion layer (a) and hardness indentations on oxidized samples Ti6Al7Nb (b) and Ti6242S (c)

\section{Discussion}

We believe it can be attributed to the following differences in behaviors of titanium alloys. Titanium alloy surface after oxidation exhibits an oxide layer which thickness was found to be different in the studied alloys. The oxide surface layer acts as a partial barrier limiting the interaction of metal with atmospheric gases at elevated temperatures. Various parameters of oxides such as phase composition, thickness and density influence the rate of diffusion processes. Between VT20 and Ti6242S alloys with a diffusion layer of the same thickness, VT20 alloy having significantly thicker oxide demonstrates lower rate of diffusion through the oxide, which probably determines the final concentration of oxygen dissolved in metal which is lower than that of Ti6242S alloy. Ti6Al7Nb alloy with the thinnest oxide and diffusion layer exhibits the minimum oxygen concentration after oxidation. The oxide diffraction lines of Ti6Al7Nb alloy are much more intense than diffraction lines of VT18U and Ti6242S alloys, which have just a slightly thicker oxide, which suggests that the density of the Ti6Al7Nb alloy oxide is higher and diffusion rate through the oxide is probably lower than in other alloys. One more parameter of the alloys which could obviously influence the oxygen concentration in the studied alloys is oxygen solubility, which, as known, depends on the alloy chemical composition [26].

In this study, we have shown a good correlation of data obtained by XRD and NMA methods. Ultimately, the difference in oxygen concentration in gas-rich layer determines the deformation behavior of the alloys [32, 33], increasing oxygen concentration in gas-rich layer leads to reduction of ductility of the oxidized alloys until completely brittle fracture in the elastic portion of load diagram. For example, VT18U and Ti6AI7Nb alloys in the oxidized condition studied herein demonstrate tensile elongation of 4.6 $\%$ and $13.2 \%$ [32] respectively due to the significant difference in oxygen concentration in gas-rich layer (difference of concentration near the oxide/metal interface $5 \mathrm{wt}$ \%).

\section{Conclusion}

Titanium alloys VT18U, VT20, Ti6Al7Nb and Ti6242S were studied after oxidation in air at $560{ }^{\circ} \mathrm{C}$ for 1000 hours. Parameters of diffusion layer on the alloy surfaces were studied by microhardness measurements, optical microscopy, X-ray diffraction analysis and nuclear microanalysis. It was determined that concentration of oxygen in diffusion layer of tested alloys after oxidation differs significantly.

The study of the effect of oxidation on the lattice parameters of metal and concentration of oxygen in diffusion layer can facilitate understanding of their effect on the a phase plasticity and mechanical properties of titanium alloys after exposure at elevated temperature.

\section{Acknowledgments}


The authors are grateful to T.E. Kurennykh from IMP UB RAS for her help in the nuclear microanalysis studies and O. Kuzmina for assistance in preparing the article.

\section{$\underline{\text { References }}$}

1. Solonina O.P., Glazunov S. G. Heat-resistant titanium alloys, Metallurgiya, Moscow (1976).

2. Blenkinsop P., Titanium-Science and Technology. 4 (1984) 2323-2338.

3. Eylon D. et al., JOM. 36 (1984) 55-62.

4. Moiseev V. N., Proc. 9th World Conf. Titanium. Russia. (1999) 48-55.

5. Donachie M. J. Titanium: a technical guide. ASM international (2000).

6. Pavlova T.V., Kashapov O.S., Nochovnaya N.A., All materials. Encyclopedic reference. 5 (2012) 8-14.

7. Merlin P., 47th AIAA aerospace sciences meeting (2009) 1522.

8. Gulbransen E.A., Andrew K.F., J. Electrochem. Soc. 96 (1949) 364-376.

9. Wasilewski H.J. and Kehl G.L., J. Inst. Met. 83 (1954) 94.

10. Shenoy R.N., Unnam J., Clark R.K., Oxid. Met. 26 (1986) 105-124.

11. Parris W.M., Bania P.J., Titanium'92: Science and technology. (1993) 153-160.

12. Geary B. et al., Titanium'95: Science and technology. (1996) 1638-1645.

13. Leyens C. et al., Metall. Mater. Trans. A. 27 (1996) 1709-1717.

14. Sansoz F., Almesallmy M., Ghonem H., Metall. Mater. Trans. A. 35 (2004) 3113-3127.

15. Kalienko M.S., Volkov A.V., Leder M.O., Zhelnina A.V.: Proc. 14th Int. Conf. Creep. (2017) 89.

16. TOPAS. v3, Karlsruhe, Germany: Bruker AXS (2005).

17. Shipatov E.T., Fast ion backscattering theory, experimental, practice. Rostov Univer. Press (1988) 160.

18. Moder N.I., Levin I.V., Vykhodets V.B., Kurennykh T.E., J. Titanium. (2003) 49-56.

19. Pilchak A.L., Porter W.J., John R., J. Mater. Sci. 47 (2012) 7235-7253.

20. Meagher E. P., G. A. Lager, The Canadian Mineralogist. 17 (1979) 77-85.

21. Garbacz H., Lewandowska M., Mater. Chem. Phys. 81 (2003) 542-547.

22. Conrad H., Prog. Mater. Sci. 26 (1981) 123-403.

23. Cabrera N., Mott N. F. Theory of the oxidation of metals, Rep. Prog. Phys. 12 (1949) 163.

24. Stringer J., Acta Metall. 8 (1960) 758-766.

25. Ignatov V. et al., Proc. 2nd World Conf. on Titanium. (1973) 2535-2544.

26. Chaze A.M., Coddet C. and Beranger G.: Proc.6th World Conf. on Titanium. (1988) 1765-1770.

27. Kalienko M.S., Volkov A.V., Zhelnina A.V., Industr. Lab. Diagnostics Mater. 84 (2018) 32-35.

28. Moroishi T., Shida Y., Nippon Kinzoku Gakkai-Shi. 42 (1978) 316-323.

29. Wiedemann K.E., Shenoy R.N., Unnam J., Metall. Trans. A. 18 (1987) 1503-1510.

30. Baillieux J., Poquillon D., Malard B. J. Appl. Cryst. 49 (2016) 175-181.

31. Dadé M. et al., Corros. Sci. 148 (2019) 379-387.

32. Kalienko M.S., Met. Sci. Heat Treat. 61 (2019) 489-494.

33. Kalienko M.S., Russ. Metall. 4 (2020) in press. 